\title{
Metalloproteinases and tissue inhibitors of metalloproteinases in exudative pleural
} effusions

\author{
D. Iglesias*, J. Alegre*, C. Alemán*, E. Ruíz*, T. Soriano*, L.l. Armadans*, \\ R.M. Segura ${ }^{+}$, A. Anglés ${ }^{+}$, J. Monasterio ${ }^{+}$and T. Fernández de Sevilla*
}

ABSTRACT: The aim of this study was to assess the expression of several metalloproteinases (MMPs) and tissue inhibitors of metalloproteinases (TIMPs) in exudative pleural effusions, and their relationship with inflammatory and fibrinolytic mediators in parapneumonic effusions.

The study included 51 parapneumonic effusions (30 empyema or complicated parapneumonic, 21 noncomplicated parapneumonic), 28 tuberculous, 30 malignant and 30 transudates. Inflammatory markers (tumour necrosis factor- $\alpha$, interleukin-8, polymorphonuclear elastase), fibrinolytic system variables (tissue plasminogen activator (PA), urokinase PA (u-PA), plasminogen activation inhibitor (PAI)-1, PAI-2), and several MMPs (MMP-1, MMP-2, MMP-8, MMP-9) and TIMPs (TIMP-1, TIMP-2) were determined by ELISA in plasma and pleural fluid.

Elevated MMP-2 and TIMP-1 concentrations were observed in all the pleural fluid samples studied. The group of empyema or complicated parapneumonic effusions showed higher MMP-1, MMP-8 and MMP-9 concentrations than the remaining exudates. There was no correlation between MMP and TIMP levels in plasma and pleural fluid in this group of effusions. In parapneumonic effusions, MMP-1, MMP-8 and MMP-9 showed a positive correlation with the inflammatory markers and with u-PA and PAI-1. Moreover, there was a relationship between MMP8 concentration in pleural fluid and pleural thickening at the end of treatment.

In conclusion, elevated metalloproteinase-1, -8 and -9 expression was found in parapneumonic pleural effusions. These metalloproteinases could be implicated in the local inflammatory response existing in this group of effusions.

KEYWORDS: Fibrinolytic system, inflammatory markers, matrix metalloproteinases, pleural effusion, tissue inhibitors of metalloproteinases

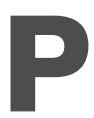

roteolytic enzymes that are responsible for breakdown of the extracellular matrix play an important role in inflammatory processes, although they have been hardly studied in relation to the pathogenesis of exudative pleural effusions [1, 2]. The metalloproteinases (MMPs) are a family of proteases containing $\geqslant 21$ members that break down virtually all the protein components of the extracellular matrix. Four subgroups of MMPs have been described according to their structure and substrate specificity: collagenases (MMP-1, MMP-8), gelatinases (MMP-2, MMP-9), stromelysins and transmembrane MMPs [3-5]. The activity of the MMPs is controlled by specific tissue inhibitors of metalloproteinases (TIMPs) [6]. Disruption of the balance between MMPs and their TIMPs is implicated in various pathological processes [7-9].
Inflammatory mechanisms and alterations in the balance of pleural fibrinolysis have been implicated in the pathophysiology of infectious pleural effusions [10]. The inflammatory response initiated by the mesothelium provokes increased vascular patency and the release of inflammatory mediators, e.g. interleukin (IL)-8 and tumour necrosis factor alpha (TNF)- $\alpha$ [11-13], thereby favouring the formation of exudative pleural effusion, invasion of the pleural space by inflammatory cells and the release of neutrophil degranulation products, such as polymorphonuclear elastase (PMN-E) [14]. The fibrinolytic system is regulated by two plasminogen activators (PAs), tissue PA (t-PA) and urokinase PA (u-PA), and by plasminogen activator inhibitor type 1 (PAI-1) and 2 (PAI-2). Loculated parapneumonic pleural effusions are associated with fibrin deposition over the pleural surface due to inhibition of the fibrinolysis system $[10,15,16]$.

\section{AFFILIATIONS}

Depts of *Internal Medicine,

\#Preventive Medicine,

"Biochemistry, and

+Haemostasis, Hospital Vall d'Hebrón, Barcelona, Spain

CORRESPONDENCE

D. Iglesias Sáenz

C/ Enric Granados $702^{\circ} 2^{\mathrm{a}}$ 08008 Barcelona

Spain

Fax: 34937921346

E-mail: 30805dis@comb.es

Received:

January 262004

Accepted after revision:

August 232004 
The aim of the current study was to study several MMPs and their TIMPs in pleural effusion, and to evaluate the association between these enzymes and inflammatory markers and elements of the fibrinolysis system in parapneumonic pleural effusion.

\section{PATIENTS AND METHODS Study population}

The study included patients $>18$ yrs old with pleural effusion, admitted to a general teaching hospital (Dept of Internal Medicine, Hospital Vall d'Hebrón, Barcelona, Spain). The study population was comprised of 139 patients with pleural effusion (60\% males; $40 \%$ females; age range: $18-94$ yrs old), including 51 parapneumonic effusions (30 empyema or complicated parapneumonic, 21 noncomplicated parapneumonic), 28 tuberculous effusions, 30 malignant effusions and 30 transudates.

Effusions were categorised as exudates or transudates according to the LIGHT et al. [17] criteria. Empyema or complicated parapneumonic effusion met one or more of the following criteria: macroscopic pus, presence of organisms on Gram stain or culture, pleural fluid $\mathrm{pH}<7.2$ or fluid glucose concentrations $<40 \mathrm{mg} \cdot \mathrm{dL}^{-1}$ (in the context of pleural effusion, these last two criteria were associated with a clinical and radiological pneumonic process) [18]. Noncomplicated parapneumonic effusions were defined as follows: patients with clinical and radiological signs consistent with pneumonia, nonpurulent pleural fluid, negative microbiological studies in pleural fluid, pleural fluid $\mathrm{pH}>7.2$ and pleural fluid glucose $>40 \mathrm{mg} \cdot \mathrm{dL}^{-1}$ [18]. Tuberculous effusion was diagnosed by a positive pleural fluid culture, caseating granulomas on pleural biopsy, or pleural fluid adenosine deaminase levels $>45 \mathrm{IU} \cdot \mathrm{L}^{-1}$ with a predominance of lymphocytes and good response to antituberculosis treatment [19]. Malignant effusions were identified by pleural fluid cytology that was positive for malignant cells or pleural histology that was diagnostic of neoplasm. Transudates were associated with heart failure.

\section{Biochemical, haemostatic and cytological methods}

Blood and pleural samples were obtained during diagnostic thoracocentesis. The Local Committee of Clinical Assays (Barcelona, Spain) approved the study.

\section{Assessment of MMPs, TIMPs and inflammatory and fibrinolytic system markers}

Blood and pleural fluid samples were collected in tubes containing 3.8\% citrate (Becton Dickinson, Le Pont de Claix, France) and the tripotassium salt of ethylene diamine tetraacetic acid (Beckton Dickinson). They were centrifuged within $1 \mathrm{~h}$ after collection at $1800 \times g$ and $4^{\circ} \mathrm{C}$. Supernatants were stored in aliquots at $-70^{\circ} \mathrm{C}$ until analysis.

The following four MMPs and two TIMPs were studied: interstitial collagenase (MMP-1), neutrophil collagenase (MMP8), gelatinase-A (MMP-2), gelatinase-B (MMP-9), TIMP-1 and TIMP-2. Determinations were carried out using an ELISA method (Amersham Pharmacia, Little Chalfont, UK). The technique recognises: total MMP-1, both free and complex forms with TIMP-1; the precursor and active forms of MMP-8, both free and complex forms with TIMP; the precursors of
MMP-2 and MMP-9, both free and complex forms with TIMP-1 and -2; and free and complex forms of the TIMPs.

TNF- $\alpha$ and IL-8 were measured by an ELISA from Innogenetics, Gent, Belgium, and from Bender Medsystems, Vienna, Austria, respectively. PMN-E was assessed with an immunoagglutination method (ECOLINE-PMN-E; Merck, Darmstadt, Germany).

The fibrinolytic system variables, u-PA, t-PA, PAI-1 antigen (Ag) and PAI-2 Ag, were measured by ELISA (TintElize; Biopool, Umea, Sweden).

\section{Radiological method}

Posteroanterior and lateral chest radiographs were performed in all patients at the time of admittance and at the end of treatment. To determine the presence of local pleural complications in the patients with parapneumonic pleural effusion, residual pleural thickening, defined as a pleural thickness $>10 \mathrm{~mm}$ on chest radiography, was evaluated. Pleural thickness at the lateral inferior wall of the affected hemithorax was measured by passing an imaginary horizontal line over the highest part of the diaphragmatic dome.

\section{Statistical analysis}

Data are presented as median (interquartile range (IQR)). Normal distribution of variables was checked using the Kolmogorov-Smirnov test. ANOVA was used to compare the distribution of variables between the different types of pleural effusion. Orthogonal contrasts were used to compare transudates $(n=30)$ with exudates $(n=109)$, infectious $(n=79)$ with malignant $(n=30)$, parapneumonic $(n=51)$ with tuberculous $(n=28)$, and empyema $(n=30)$ with noncomplicated parapneumonic $(n=21)$. In this analysis, logarithmic transformation was applied to variables that showed non-normal distribution. Differences between groups are presented as mean (fold) differences with $95 \%$ confidence intervals (CI). Comparison of plasma and pleural fluid levels of MMPs and TIMPS was performed with Wilcoxon's nonparametric test. The Spearman coefficient of correlation was used to study the association of MMPs and TIMPs with inflammatory and fibrinolysis system markers in parapneumonic pleural effusion. The nonparametric Mann-Whitney U-test was applied to study pleural fluid levels of MMPs and TIMPs in relation to pleural thickening in patients with parapneumonic pleural effusion.

\section{RESULTS}

\section{MMPs and TIMPs in pleural fluid}

Table 1 shows the median (IQR) of pleural fluid MMP and TIMP concentrations in the various types of pleural effusions studied. Elevated MMP-2 and TIMP-1 levels were found in all the pleural fluid samples studied (exudates and transudates).

In the study of exudative effusions, the group of empyema or complicated parapneumonic effusions showed the highest concentrations of MMP-1, MMP-8 and MMP-9, with significant differences relative to the remaining exudates and noncomplicated parapneumonic effusions (MMP-1: mean difference $(95 \%$ CI) 2.18 (1.40-3.41), $\mathrm{p}=0.001$; MMP-8: 10.32 (3.65-29.18), $\mathrm{p}<0.001$, fig. 1; MMP-9: 4.24 (1.62-11.09), $\mathrm{p}=0.005)$. Nevertheless, MMP-2 concentrations were lower in this group $(p<0.001)$. Finally, infectious effusions showed higher levels 
TABLE 1 Metalloproteinases (MMPs) and tissue inhibitors of metalloproteinases (TIMPS) in pleural fluid, according to the aetiology of pleural effusions

Empyema
Noncomplicated

parapneumonic
Tuberculous

Malignant

Transudate

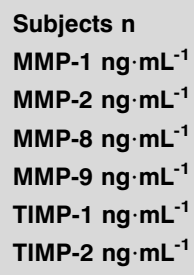

Subjects $\mathbf{n}$

30

$11.8(6.8-19.9)$

1170.6 (231-1342)

1302.5 (275.3-1518.0)

292.9 (174.4-415.6)

24000.0 (6582-32625)

$81.6(52.6-130.1)$
21

$4.8(3.2-6.9)$

$1741.6(1601-2128)$

$59.4(10.7-295.8)$

$49.2(9.2-196.4)$

$23600.0(17500-30950)$

$66.0(52.2-87.5)$
28

$5.2(3.4-7.6)$

1899.7 (1679-2625)

$85.2(17.2-121.2)$

$69.7(44.7-137.1)$

32850.0 (22725-36325)

$91.8(70.2-112.3)$
30

$3.8(2.1-5.1)$

30

1578.3 (1331-2085)

$3.5(1.7-12.0)$

$8.1(5.0-32.5)$

$17300.0(13200-22100)$

101.6 (77.4-139.1)
$1.8(1.7-2.5)$

1257.8 (1078-1493)

$1.2(0.5-2.5)$

$4.0(2.2-7.8)$

$12100.0(11475-13675)$

59.6 (46.1-98.5)

Data are presented as $\mathrm{n}$ and median (interquartile range)

of TIMP-1 than neoplastic effusions (TIMP-1: 1.01 (0.63-1.76); $\mathrm{p}<0.001$ ).

\section{MMPs and TIMPs in plasma and pleural fluid in the group of empyema or complicated parapneumonic effusions}

Concentrations of MMP-1, MMP-2, MMP-8 and TIMP-1 in pleural fluid were significantly higher than those in plasma ( $\mathrm{p}<0.001$; plasma median (IQR) MMP-1: $5.4 \mathrm{ng} \cdot \mathrm{mL}^{-1}$ (3.9-7.1); MMP-2: $551.5 \mathrm{ng} \cdot \mathrm{mL}^{-1}$ (369-663); MMP-8: $30.7 \mathrm{ng} \cdot \mathrm{mL}^{-1}(19-$ 55); TIMP-1: $\left.1,440 \mathrm{ng} \cdot \mathrm{mL}^{-1}(1230-1824)\right)$. No correlation was found between plasma and pleural fluid levels of MMPs and TIMPs.

\section{Inflammatory and fibrinolytic markers in pleural fluid}

Table 2 displays the median (IQR) of pleural fluid inflammatory and fibrinolytic markers, total cell count and percentage of neutrophils, according to the aetiology of pleural effusions. The exudates presented higher concentrations of inflammatory and fibrinolysis system markers than the transudates $(p<0.001)$, except for $t-P A$, which was lower in the group of

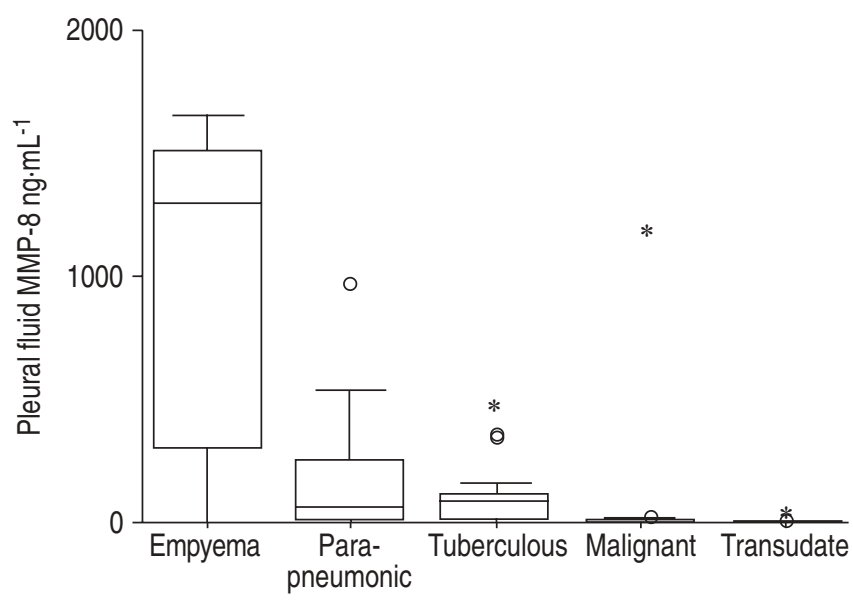

FIGURE 1. Pleural fluid concentrations of metalloproteinase (MMP)-8 according to the aetiology of pleural effusions. Data are presented as box-plots, where the horizontal line represents the median. Parapneumonic: noncomplicated parapneumonic pleural effusions. *: extremes, i.e. values more than three box lengths from either the 25th or the 75th percentile; $\bigcirc$ : outliers, i.e. values more than 1.5 box lengths from either the 25th or the 75th percentile. $p<0.001$ empyema or complicated parapneumonic effusions. This last group showed higher levels of IL-8 and PMN-E than the remaining infectious effusions $(p<0.001)$, whereas TNF- $\alpha$ was highest in the tuberculous effusions $(p<0.05)$. PAI-1 Ag showed higher values in empyema and tuberculous effusions than in noncomplicated parapneumonic effusion $(\mathrm{p}<0.001)$. Leukocyte and neutrophil counts were higher in empyema than the remaining groups studied.

\section{Association of MMPs and TIMPs with inflammatory and fibrinolysis markers in parapneumonic effusions}

Table 3 shows the correlation of MMPs and TIMPs with inflammatory and fibrinolysis system markers in pleural fluid. MMP-1, MMP-8 and MMP-9 correlated positively with the inflammatory markers IL- 8, PMN-E and TNF- $\alpha$, and with the fibrinolytic system markers u-PA and PAI-1 Ag. MMP-2 correlated negatively with IL-8, PMN-E and PAI-1 Ag.

\section{Clinical and radiological evolution of parapneumonic effusions}

Tube thoracostomy and drainage was performed in 25 patients $(83.3 \%)$ with empyema or complicated parapneumonic effusion. Among the patients who did not undergo drainage in this group, all had nonpurulent effusions, one was a terminal patient who died during hospitalisation, three underwent thoracocentesis without recurrence of the effusion, and chest drainage was attempted in another without success, but posterior clinical and radiological evolution with antibiotic treatment was favourable. In the group of noncomplicated parapneumonic effusions, progression of the effusion and presence of a loculation requiring drainage was observed in the clinical evolution of three patients. All had elevated pleural fluid lactate dehydrogenase values, a parameter that was not included in the differentiation between complicated and noncomplicated parapneumonic effusions. Intrapleural fibrinolytic treatment was added in 15 patients with empyema or complicated parapneumonic effusions and in the three noncomplicated patients. Two empyema cases (6.7\%) required pleural decortication. Seven patients with empyema died during hospitalisation: four deaths were related to pleural sepsis and three to the underlying pathologies.

Pleural thickening was detected at the end of treatment in 14 patients $(27.5 \%)$. Pleural fluid concentrations of MMP-8 were 
TABLE 2 Inflammatory and fibrinolytic markers, total cell count and percentage of neutrophils in pleural fluid, according to aetiology of the pleural effusion

Subjects $\mathbf{n}$

PMN-E $\mu \mathrm{g} \cdot \mathrm{L}^{-1}$

IL-8 pg. $\mathrm{mL}^{-1}$

TNF- $\alpha \mathrm{pg} \cdot \mathrm{mL}^{-1}$

t-PA $\mathrm{ng} \cdot \mathrm{mL}^{-1}$

u-PA $\mathrm{ng} \cdot \mathrm{mL}^{-1}$

PAl-1 Ag $\mathrm{ng} \cdot \mathrm{mL}^{-1}$

PAl-2 Ag ng $\cdot \mathrm{mL}^{-1}$

Total cell count $\times 10^{3} \cdot \mathrm{L}^{-1}$

Neutrophils $\times 10^{3} \cdot \mu \mathrm{L}^{-1}$
Empyema

30

5578.0 (2524-9549)

18114.0 (1139-55515)

$56.5(15.8-215.5)$

$5.4(2.1-15.8)$

$2.5(1.5-4.5)$

1772.2 (979.6-1943.3)

74.9 (41.0-132.6)

12000 (2425-38850)

10080 (1890-32559)
Noncomplicated
parapneumonic

21

Tuberculous

$181.5(47.5-640.0)$

$17.0(3.5-496.5)$

$24.0(12.5-44.5)$

$21.3(9.1-28.3)$

$0.8(0.6-1.1)$

104.8 (34.0-863.5)

48.6 (11.6-110.6)

4300 (1100-9300)

938 (237-6356)
28

28
$258.3(111.8-605.5)$
$75.0(48.8-203.0)$
$150.5(90.5-242.5)$
$13.4(8.0-23.0)$
$1.4(1.1-2.3)$
$1500.1(963.5-1769.8)$
$58.1(20.9-89.7)$
$2450(975-5375)$
$282(97-1047)$

Malignant

30

$20.0(10.8-46.8)$
$105.0(18.8-253.3)$
$14.0(8.8-22.5)$
$20.4(12.5-29.7)$
$1.9(1.0-4.2)$
$213.0(52.9-567.3)$
$26.7(10.1-35.3)$
$1400(1050-3425)$
$198(68-465)$

30

$8.0(4.8-59.3)$

$3.0(1.0-11.3)$

$7.0(3.8-10.3)$

$9.9(6.8-12.7)$

$0.4(0.2-0.5)$

15.5 (11.3-28.8)

6.7 (3.8-16.3)

450 (200-913)

40 (11-199)

Data are presented as $n$ and median (interquartile range). PMN-E: polymorphonuclear elastase; IL: interleukin; TNF: tumour necrosis factor; t-PA: tissue plasminogen activator; u-PA: urokinase plasminogen activator; PAl: plasminogen activation inhibitor; Ag: antigen.

higher in the patients with pleural thickening at the end of the treatment than in the patients that did not present this complication (median (IQR): $1033.6 \mathrm{ng} \cdot \mathrm{mL}^{-1}$ (330-1518), $205.5 \mathrm{ng} \cdot \mathrm{mL}^{-1}(26-1236)$, respectively; $\left.\mathrm{p}=0.047\right)$.

\section{DISCUSSION}

Alterations in the balance of the protease and antiprotease systems, which regulate proteolysis, breakdown, remodelling and integrity of the extracellular matrix, are known to play an important role in inflammatory processes. This study assesses several MMPs and TIMPs in pleural fluid of different aetiologies. Elevated levels of MMP-2 and TIMP-1 were observed in all of the pleural effusions studied. The novel findings of this study include the very high concentrations of MMP-1, MMP-9 and, particularly, MMP-8 in the group of empyema or complicated parapneumonic effusions, and the close positive correlation between these enzymes and the inflammatory markers and PAI-1. Moreover, in parapneumonic effusions, MMP-8 was related to the presence of pleural thickening at the end of treatment.

\begin{tabular}{|c|c|c|c|c|}
\hline \multirow[t]{2}{*}{ TABLE 3} & \multicolumn{4}{|c|}{$\begin{array}{l}\text { Correlations (r-values) between } \\
\text { metalloproteinases (MMPs) and inflammatory } \\
\text { and fibrinolytic markers in pleural fluid in } \\
\text { parapneumonic effusions } \#\end{array}$} \\
\hline & MMP-1 & MMP-2 & MMP-8 & MMP-9 \\
\hline PMN-E & $0.433^{*}$ & $-0.726^{\star *}$ & $0.677^{\star \star}$ & $0.434^{\star *}$ \\
\hline IL-8 & $0.416^{\star}$ & $-0.673^{\star \star}$ & $0.629^{\star \star}$ & $0.368^{\star}$ \\
\hline TNF- $\alpha$ & $0.398^{\star}$ & & $0.544^{\star \star}$ & $0.548^{\star *}$ \\
\hline u-PA & $0.498^{* *}$ & & $0.726^{\star *}$ & $0.481^{* *}$ \\
\hline PAl-1 Ag & $0.532^{* *}$ & $-0.443^{\star *}$ & $0.805^{\star *}$ & 0.739 ** \\
\hline
\end{tabular}

PMN-E: polymorphonuclear elastase; IL: interleukin; TNF: tumour necrosis factor; u-PA: urokinase plasminogen activator; PAl: plasminogen activation inhibitor; Ag: antigen. *: $p<0.01$; ${ }^{*}: p<0.001$; ${ }^{*}: n=51$.

\section{MMPs and TIMPs in pleural effusion}

With respect to MMP-2, the constant presence of this enzyme in pleural effusions was attributed to its constitutive expression by mesothelial cells. Exudates presented higher levels than transudates, suggesting that other cells with important roles in the pathogenesis of these effusions, such as mononuclear cells, might collaborate in MMP-2 release [20]. Moreover, this circumstance would explain the relatively low levels of MMP-2 in empyema, given the clear neutrophilic predominance in these effusions.

It is suggested that the prevalence of TIMP-1 over TIMP-2 in the pleural fluids studied would also correspond to constitutive expression of this peptide by mesothelial cells, and that the significantly increased values in infectious pleural effusions would be due to stimulation by inflammatory mediators.

MMP-1 levels were elevated in empyema and complicated parapneumonic effusions, and it is believed that this finding might be related to the release of this enzyme by mesothelial cells stimulated by inflammatory mediators.

Finally, MMP-8 and MMP-9 concentrations were significantly elevated in infectious pleural effusions, particularly empyema. This fact may be related with the intense neutrophilic response observed in this group of pleural effusions, since these MMPs are stored in the neutrophil granules.

It was also found that concentrations of MMP-8 in pleural fluid were five-fold greater in parapneumonic pleural effusion patients showing pleural thickening at the end of treatment. This evidence seems to implicate MMP-8 in the development of late pleural complications in this group of pleural effusions.

There are few studies in the literature on the role of the MMPs in pleural effusion. The authors of two previous reports did not find significant differences in the levels of MMP-1, MMP-2 and TIMP-1 in several types of pleural fluids [1, 2]. Thus, they considered their expression in pleural fluid to be a constitutive characteristic of the resident mesothelial cells [1, 2]. The in vitro studies by MARSHALL et al. [21] demonstrated that mesothelial cells constitutively express MMP-2 and TIMP. 


\section{MMPs and TIMPs in plasma and pleural fluid in empyema and complicated parapneumonic pleural effusions}

In this group of effusions, higher levels of MMPs and TIMPs were found in pleural fluid than in plasma, and no correlation was found between the concentrations in the two samples. These findings suggest a compartmentalisation of these enzymes in the pleural space.

\section{Inflammatory and fibrinolytic markers in pleural fluid}

In the present study, inflammatory markers and PAIs were highly elevated in parapneumonic effusions. These findings concur with results from other studies and indicate that in infectious pleural effusions, particularly empyema, there is an intense inflammatory response and inhibition of fibrinolysis that would favour fibrin deposition [10, 12-16].

\section{Association of MMPs and TIMPs with inflammatory and fibrinolytic markers in parapneumonic effusions}

The current results in parapneumonic effusions showed a close positive correlation of MMP-1, MMP-8 and MMP-9 with the three inflammatory markers studied. It seems, therefore, that IL-8 and TNF- $\alpha$ favour chemotaxis and degranulation of neutrophils in the pleural space. This fact would lead to increased levels of MMP-8, MMP-9 and PMN-E [12] and, at the same time, stimulate MMP-1 release by mesothelial cells. It was also found that MMP-1, MMP-8 and MMP-9 correlated positively with u-PA and PAI-1 Ag in this group of effusions. Assuming that there is an intense neutrophilic inflammatory response and inhibition of the fibrinolytic pathway in parapneumonic effusions, the current authors conclude that these three metalloproteinases would play a role in the inflammatory scenery of infectious pleural effusions.

In addition, it was found that MMP-2 correlated negatively with PMN-E, IL-8 and PAI-1 in this group of infectious effusions. Several studies coincide in the role of plasmin and the fibrinolysis pathway in the activation of MMP-2 [3, 22]. Thus, the significantly lower levels of MMP-2 in parapneumonic effusions could be explained by the fact that the action of plasminogen inhibitors blocking the fibrinolytic pathway predominates in these effusions. It seems clear that the inflammatory state has repercussions on MMP-2 expression by mesothelial cells.

There are no studies assessing the association of MMPs and TIMPs with inflammatory and fibrinolysis system markers in pleural effusion. In keeping with the current results, studies on the neutrophil inflammatory response have shown a positive association between MMP-9 and the proinflammatory cytokines IL- 8 and TNF- $\alpha$ [8, 9]. Along this line, BLÄSER et al. [23] reported that MMP-8 and MMP-9 correlate with u-PA and PAI-2 Ag in the synovial fluid of patients undergoing chemical synoviorthesis.

Based on the results from the present study, the current authors suggest that the response of the pleural serosa to bacterial injury, with triggering of the inflammatory cascade and inhibition of fibrinolysis, provokes alterations in the balance between the metalloproteinases and their inhibitors. Metalloproteinase-1, -8 and -9 are intimately implicated in the pleural inflammatory response. Breakdown of the extracellular matrix by these three enzymes would favour chemotaxis of the inflammatory cells and fibroblasts towards the pleural space, perpetuating the inflammatory response and the subsequent local complications of pachypleuritis and pleural fibrosis.

\section{ACKNOWLEDGEMENTS}

The authors would like to thank the nursing supervisor, M. Murias, and the nurses, I. Vera and M.J. Gascón, for their participation. The authors also thank C. Carvallo for linguistic advice.

\section{REFERENCES}

1 Hurewitz AN, Zucker S, Mancuso P, et al. Human pleural effusions are rich in matrix metalloproteinases. Chest 1992; 102: 1808-1814

2 Eickelberg O, Sommerfeld CO, Wyser C, et al. MMP and TIMP expression pattern in pleural effusions of different origins. Am J Respir Crit Care Med 1997; 156: 1987-1992.

3 Woessner JF. Matrix metalloproteinases and their inhibitors in connective tissue remodeling. FASEB J 1991; 5: 2145-2154.

4 Birkedal-Hansen H, Moore WGI, Bodden MK, et al. Matrix metalloproteinases: a review. Crit Rev Oral Biol Med 1993; 4: 197-250.

5 Murphy G, Docherty AJP. The matrix metalloproteinases and their inhibitors. Am J Respir Cell Mol Biol 1992; 7 $120-125$.

6 Gomez DE, Alonso DF, Yoshiji H, Thorgeirsson UP. Tissue inhibitors of metalloproteinases: structure, regulation and biological functions. Eur J Cell Biol 1997; 74: 111-122.

7 Ray JM, Stetler-Stevenson WG. The role of matrix metalloproteinases and their inhibitors in tumour invasion, metastasis and angiogenesis. Eur Respir J 1994; 7: 2062-2072.

8 Pugin J, Widmer MC, Kossodo S, Liang CM, Preas HL 2nd Suffredini AF. Human neutrophils secrete gelatinase $\mathrm{B}$ in vitro and in vivo in response to endotoxin and proinflammatory mediators. Am J Respir Cell Mol Biol 1999; 20: 458-464.

9 Leppert D, Leib SL, Grygar C, Miller KM, Schaad UB Hollander GA. Matrix metalloproteinase (MMP)-8 and MMP-9 in cerebrospinal fluid during bacterial meningitis: association with blood-brain barrier damage and neurological sequelae. Clin Infect Dis 2000; 31: 80-84.

10 Philip-Joét F, Alessi MC, Philip-Joét C, et al. Fibrinolytic and inflammatory processes in pleural effusions. Eur Respir 1995; 8: 1352-1356.

11 Antony VB, Mohammed K. Pathophysiology of pleural space infections. Semin Respir Infect 1999; 14: 9-17.

12 Segura RM, Alegre J, Varela E, et al. Interleukin-8 and markers of neutrophil degranulation in pleural effusions. Am I Respir Crit Care Med 1998; 157: 1565-1572.

13 Strieter RM, Lukacs NW, Standiford TJ, Kunkel SL. Cytokines. 2. Cytokines and lung inflammation mechanisms of neutrophil recruitment to the lung. Thorax 1993; 48: 765-769.

14 Alegre J, Suriñach JM, Varela E, et al. Diagnostic accuracy of pleural fluid polymorphonuclear elastase in the differentiation between pyogenic bacterial infectious and noninfectious pleural effusions. Respiration 2000; 67: 426-432. 
15 Idell S, Girard W, Koenig K, McLarty J, Fait DS. Abnormalities of pathways of fibrin turnover in the human pleural space. Am Rev Respir Dis 1991; 144: 187-194.

16 Agrenius V, Chmielewska J, Widström O, Blomback M. Pleural fibrinolytic activity is decreased in inflammation as demonstrated in quinacrine pleurodesis treatment of malignant pleural effusion. Am Rev Respir Dis 1989; 140: 1381-1385.

17 Light RW, MacGregor MI, Luchsinger PC, Ball WC Jr. Pleural effusion: the diagnostic separation of transudates and exudates. Ann Intern Med 1972; 77: 507-513.

18 Light RW, Girard WM, Jenkinson SG, George RB. Parapneumonic effusions. Am J Med 1980; 69: 507-512.

19 Ocaña I, Martínez Vázquez JM, Segura RM, Fernandez-DeSevilla T, Capdevila JA. Adenosine deaminase in pleural fluid. Test for diagnosis of tuberculous pleural effusion. Chest 1983; 84: 51-53.
20 Segura-Valdez L, Pardo A, Gaxiola M, Uhal BD, Becerril C, Selman M. Upregulation of gelatinases A and B, collagenases 1 and 2, and increased parenchymal cell death in COPD. Chest 2000; 117: 684-694.

21 Marshall BC, Santana A, Xu QP, et al. Metalloproteinases and tissue inhibitor of metalloproteinases in mesothelial cells. Cellular differentiation influences expression. J Clin Invest 1993; 91: 1792-1799.

22 Mazzieri R, Masiero L, Zanetta L, et al. Control of type IV collagenase activity by components of the urokinaseplasmin system: a regulatory mechanism with cell bound reactants. EMBO J 1997; 16: 2319-2332.

23 Bläser J, Triebel $\mathrm{S}$, Maasjosthusmann $\mathrm{U}$, et al. Determination of metalloproteinases, plasminogen activators and their inhibitors in the synovial fluids of patients with rheumatoid arthritis during chemical synoviorthesis. Clin Chim Acta 1996; 244: 17-33. 